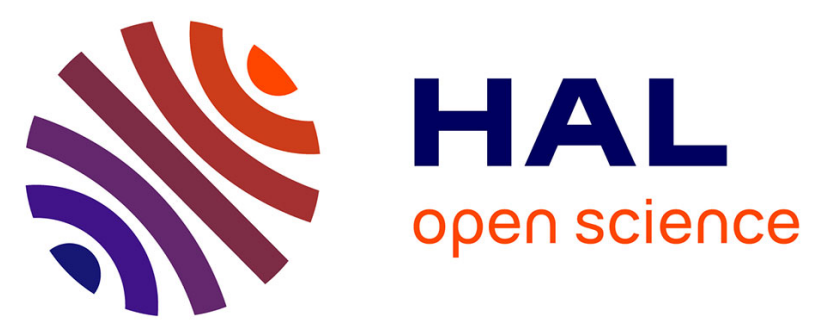

\title{
Implantation du sphincter artificiel AMS 800® chez la femme par cœlioscopie robot-assistée: expérience monocentrique
}

\author{
P. Callerot, M.-A. Perrouin-Verbe, M. Thoulouzan, A. Valeri, G. Fournier
}

\section{- To cite this version:}

P. Callerot, M.-A. Perrouin-Verbe, M. Thoulouzan, A. Valeri, G. Fournier. Implantation du sphincter artificiel AMS $800 \AA$ chez la femme par cœlioscopie robot-assistée: expérience monocentrique. Progrès en Urologie, 2019, 29, pp.246 - 252. 10.1016/j.purol.2018.12.007 . hal-03486235

\section{HAL Id: hal-03486235 \\ https://hal.science/hal-03486235}

Submitted on 20 Dec 2021

HAL is a multi-disciplinary open access archive for the deposit and dissemination of scientific research documents, whether they are published or not. The documents may come from teaching and research institutions in France or abroad, or from public or private research centers.
L'archive ouverte pluridisciplinaire HAL, est destinée au dépôt et à la diffusion de documents scientifiques de niveau recherche, publiés ou non, émanant des établissements d'enseignement et de recherche français ou étrangers, des laboratoires publics ou privés.

\section{다)(1) $(5$}

Distributed under a Creative Commons Attribution - NonCommerciall 4.0 International 
Version of Record: https://www.sciencedirect.com/science/article/pii/S1166708718306869

Manuscript_9cc5c0e9d0889ece5f7829ce8e724bbc

\section{Implantation du sphincter artificiel AMS $800 \AA$ chez la femme par cœlioscopie} robot-assistée : expérience monocentrique.

Pierre Callerot, Marie-Aimée Perrouin-Verbe, Maxime Thoulouzan, Antoine Valeri,

Georges Fournier.

Service d'urologie Centre Hospitalier Universitaire de Brest.

Correspondance :

Dr Pierre CALLEROT, Assistant spécialiste, service d'urologie du CHU de BREST, Cavale Blanche, boulevard Tanguy Prigent 29200 BREST

pierrecallerot@yahoo.fr 


\section{Introduction}

Le sphincter urinaire artificiel (SUA) est un des traitements de référence de l'incontinence urinaire d'effort féminine par insuffisance sphinctérienne et est indiqué le plus souvent après échec d'autres traitements chirurgicaux (1). C'est une procédure techniquement difficile à réaliser chez ces patientes, dont la région du col vésical est souvent remaniée par des adhérences liées aux antécédents chirurgicaux et la survenue de plaies, vaginales ou urétro-vésicales, lors de la dissection de cet espace met en péril l'implantation du matériel (2). La procédure d'implantation par voie ouverte, a montré de bons résultats en matière de continence $(3,4)$, ainsi que la voie cœlioscopique, rapportée pour la première fois par Nginkeu et al. (5). Nous avons décrit notre technique d'implantation par cœlioscopie robot-assistée et rapporté les résultats initiaux en 2014 chez 6 patientes primo-implantées (6). Dans cet article nous rapportons les résultats de cette technique, en matière de complication et de continence, pour 14 primo-implantations, et 3 révisions de SUA.

\section{Matériel et méthodes}

\section{$\underline{\text { Patientes }}$}

Entre 2012 et 2017, étaient incluses consécutivement toutes les patientes ayant eu une implantation ou une révision de SUA dans notre centre.

Les indications opératoires étaient une incontinence urinaire d'effort (IUE) avec insuffisance sphinctérienne (IS) (7). A l'examen clinique, toutes les patientes avaient des fuites à la toux, une absence de mobilité cervico-urétrale et un test de soutènement de 
l'urètre moyen négatif. Une incontinence urinaire mixte n'était pas un critère d'exclusion.

Le bilan pré-opératoire, comportait un interrogatoire précisant le nombre de protections par jour, les antécédents chirurgicaux pour incontinence, prolapsus ou autre chirurgie pelvienne. L 'indice de masse corporelle était calculé et une évaluation cognitive et motrice était réalisée afin de s'assurer de la bonne manipulation de la pompe par la patiente. Ainsi, le côté de l'implantation de la pompe était déterminé. L'examen clinique en position gynécologique comportait la recherche d'une hypermobilité cervico-urétrale, de fuites à la toux à vessie pleine, et en cas de fuites leur disparition ou non par les manœuvres de soutènement de l'urètre moyen. Un prolapsus était systématiquement recherché. Lors du bilan urodynamique, une sphinctérométrie et une cystomanométrie étaient systématiquement réalisées. Enfin une information détaillée sur l'implantation du SUA par laparoscopie avec assistance robotique était donnée et un consentement oral obtenu.

En cas de révision de sphincter, le bilan préopératoire comportait également un bilan radiologique qui confirmait une panne mécanique par l'absence de liquide de remplissage dans le réservoir. Une cystoscopie systématique vérifiait l'absence d'érosion cervico-urétrale par la manchette.

\section{Technique chirurgicale}

Pour les primo-implantations, la technique chirurgicale était celle décrite dans notre série de 2014 (6). Depuis 2012, seule la voie coelioscopique robot-assistée est pratiquée dans cette indication, par un même opérateur, expert en chirurgie robotique, assisté 
d'un interne. Les urines étaient stériles. Une antibioprophylaxie était systématique par céphalosporine de 2ème génération.

La potence du robot (Da Vinci ® Si 4 bras) était positionnée latéralement, en dehors de la jambe droite afin de permettre un accès au vagin pour l'assistant durant la procédure. Sous anesthésie générale et après mise en place d'une sonde vésicale $14 \mathrm{ch}$, la patiente est installée en décubitus dorsal, jambes fléchies à $45^{\circ}$, et en Trendelenburg. Une optique $0^{\circ}$ et cinq trocarts étaient utilisés. Occasionnellement, un sixième trocart était implanté en sus ombilical pour faciliter l'exposition (Figure 1).

En cas de cure de prolapsus simultané à la mise en place du SUA, la promontofixation était réalisée en premier.

Le remplissage vésical par 120 cc de sérum permettait de repérer la convexité du dôme vésical et d'aborder la face antérieure de la vessie par une incision arciforme convexe vers le haut en restant en permanence au contact de l'arche pubienne. En cas d'intervention de Burch, de suspension aponévrotique ou de TVT préalable, il était habituel de sectionner la bandelette et /ou les fils lors de la dissection latérale.

La dissection se prolongeait antérieurement jusqu'au col vésical et latéralement jusqu’à la prise de contact avec le fascia endopelvien de chaque côté.

Afin de faciliter le repérage de la face antérieure du vagin, une bougie de Hégar était introduite dans le vagin et dirigée latéralement vers les culs de sac vaginaux antérieurs. Les instruments articulés (ciseaux monopolaires, pince Prograsp®, pince bipolaire) permettaient de disséquer à angle droit, suivant ainsi la direction de dehors en dedans en arrière du col d'un coté à l'autre (Figure 2). En cas de difficultés de dissection en arrière du col vésical, nous réalisions une ouverture longitudinale du dôme vésical, à distance du col, pour contrôler visuellement, par voie endovésicale, la dissection. Dans 
ce cas, après création de l'espace rétrocervical, la vessie était refermée par un surjet de fil à résorption lente et son étanchéité vérifiée.

Ensuite le mesureur permettait de déterminer la circonférence du col. La manchette était introduite par le trocart de $12 \mathrm{~mm}$ de l'assistant puis positionnée en péricervical et clipsée. Une incision sus pubienne de $3 \mathrm{~cm}$ latéralisée du côté de l'emplacement de la future pompe dans la grande lèvre était effectuée. Par cette incision le ballon de régulation des pressions (61/70 cm H20) était introduit en para vésical puis rempli sous contrôle de la vue avec du sérum physiologique. Le péritoine était alors refermé pour extrapéritoniser la manchette et le ballon. A ce stade le robot était désolidarisé des instruments, le pneumopéritoine était exsufflé et les orifices de trocart refermés. La mise en place de la pompe dans la grande lèvre du coté choisi, la connexion des tubulures et la désactivation du sphincter suivaient les étapes habituelles.

En cas de révision, l'ouverture de l'espace de Retzius permettait de repérer les tubulures destinées au réservoir et à la manchette qui étaient ensuite libérées à l'aide des ciseaux monopolaires. De proche en proche, le réservoir puis la manchette, étaient identifiés. L'ablation de la manchette péricervicale était effectuée après libération de son hémicirconférence antérieure. Le mesureur était alors solidarisé à une des extrémités de la manchette par un fil permettant de remplacer la manchette par le mesureur sans perdre le trajet de la dissection péricervico-urétrale. La manchette était retirée par le trocart de $12 \mathrm{~mm}$. Après mesure de la circonférence péricervicale une nouvelle manchette était introduite par le trocart de $12 \mathrm{~mm}$ et son extrémité était solidarisée au mesureur puis glissée autour du col vésical et enfin clipsée. La mise en place du nouveau réservoir et l'ablation du précédent étaient effectuées par une incision de $3 \mathrm{~cm}$ sus pubienne par 
laquelle les tubulures étaient ensuite extériorisées puis connectées en dehors de la cavité abdominale comme dans la technique décrite précédemment.

\section{Critères d'évaluation}

Les paramètres péri-opératoires incluaient la durée opératoire, les complications per et post opératoire, la durée de sondage et d'hospitalisation. L'évaluation à distance comportait une visite post opératoire avec l'activation du sphincter à 6 semaines, puis une consultation à 3 mois, 6 mois et 1 an, puis annuellement. Étaient recherchés des troubles mictionnels de novo (dysurie, syndrome d'hyperactivité vésicale), l'existence de complications infectieuses et une difficulté éventuelle de manipulation de la pompe. Une débitmétrie avec mesure de résidu post mictionnel était effectuée. La patiente était considérée continente en l'absence de protection.

\section{Résultats}

\section{Patientes}

Dix-sept patientes ont eu une implantation de SUA, 14 primo-implantations (PI), 3 révisions (R). Les caractéristiques détaillées des patientes sont présentées dans les tableaux 1a et 1b. Aucune patiente n'avait d'antécédent d'irradiation pelvienne. 


\section{Groupe Primo-implantation (tableau 1a)}

Douze patientes avaient au moins un antécédent de chirurgie de l'incontinence. Une patiente était sondée, à cause de fuites permanentes, après retrait d'un précèdent SUA, explanté 1 an avant l'inclusion dans l'étude (Patiente 4). Une patiente avait une vessie neurologique, acontractile, avec insuffisance sphinctérienne, aux autosondages intermittents propres (Patiente 5), secondairement à une ischémie médullaire, La patiente 14 avait une vessie hypocontractile non neurologique, les autres patientes avaient toutes une vessie normocompliante et normocontractile. Quatre patientes on fait l'objet d'une promontofixation dans le même temps opératoire (patientes 1,10,12 et 13).

\section{Groupe révision (tableau 1b)}

Les 3 patientes ont eu un remplacement de SUA en raison d'une panne mécanique, par fuite du liquide de remplissage. Chaque patiente avait pour antécédent une promontofixation et une colposuspension selon Burch, mais aucune n'avait eu de bandelette sous urétrale. La patiente 3 a reçu un premier sphincter qui a été explanté pour infection, reposé à distance, puis remplacé lors de cette étude après 14 ans de fonctionnement. Par ailleurs, la patiente 1, avait une hyperactivité détrusorienne.

\section{Intervention et suites}

\section{Groupe Primo-implantation (tableau 2)}


L'intervention durait en moyenne $205 \pm 34$ min comprenant un temps de dissection du col de $46 \pm 21 \mathrm{~min}$. En cas de promontofixation associée, la durée moyenne d'intervention était allongée en moyenne de 38 min. Onze cystotomies $(78,6 \%)$ ont été pratiquées pour faciliter la dissection cervicale. Les durées de sondage et d'hospitalisation étaient respectivement de : 5,9 $\pm 2,1$ et 6,6 $\pm 1,5$ jours. Une plaie vaginale a été observée, à distance du site d'implantation cervical (Patiente 14), et suturée au Vlock® avant la dissection péricervicale. Aucune plaie urétrale ou vésicale n’a été observée.

Trois complications post opératoires sont survenues : un hématome de la grande lèvre spontanément résolutif et deux rétentions urinaires, dont une fébrile dans les suites de la réactivation, résolutive sous antibiothérapie seule (Patiente 8).

A l'issue d'un suivi moyen de $24,6 \pm 18,4$ mois, le taux de continence totale était de $86 \%$, et 4 patientes présentent une hyperactivité vésicale de novo, contrôlée par anticholinergiques (patientes 9, 10 et 12) ou neurostimulation tibiale (patiente 3 ). Toutes les patientes, portent encore leur SUA et l'utilisent correctement.

\section{Groupe révision (tableau 3)}

L’intervention durait en moyenne $112 \pm 8 \mathrm{~min}$. Les durées de sondage et d'hospitalisation étaient respectivement de 4,3 \pm 4 et $7 \pm 3,6$ jours.

Une patiente a présenté un syndrome occlusif résolutif sous traitement médical (patiente 3).

A l'issue d'un suivi moyen de 58,15 $\pm 5,2$ mois, la continence totale était de $100 \%$. Ici aussi, les 3 SUA sont encore en place et correctement utilisés. 


\section{Discussion}

La technique opératoire que nous présentons reprend les mêmes étapes que celles développées dans la laparoscopie conventionnelle. L'intérêt des pinces robotisées à 7 degrés de liberté et la vision en 3D, procurés par l'assistance robotique, apporte de meilleures conditions à la réalisation de la dissection péricervicale. En effet cette phase est la plus pourvoyeuse de complications par risque de brèche vésico-urétrale, ou vaginale, qui peuvent compromettre l'implantation et la survie du matériel (explantation, érosion) $(3,4,8)$. Cette procédure est techniquement difficile et doit être menée par un chirurgien expérimenté.

Nous rapportons un cas de plaie vaginale (7\%) à distance du site d'implantation, chez une patiente primo-implanté, avec des suites simples à plus d'un an de recul. Ce résultat se place dans les 10,4 à 14\% de plaies retrouvées dans les séries de voies ouvertes $(3,4)$.

Concernant la laparoscopie, l' équipe de Mandron rapporte une plaie vaginale (2\%), sans conséquence ultérieure, mais 5 explantations ont été rapportées pour infection (2\%) ou érosion vaginale ou urétrale (8\%) (8). Roupret et al. rapportaient 4 plaies (33\%), dont 1 a mené à l'abandon de la procédure, les 3 autres a une conversion en laparotomie (9). De la même manière, Trolliet et al. rapportaient une plaie vaginale, passée inaperçue en peropératoire, menant à l'explantation précoce du matériel (J1), ainsi que 5 plaies vésicales (19\%) (10). 
Depuis la première publication d'une implantation robot-assistée, qui rapportait de bons résultats à court terme (6), deux autre centres ont rapporté des taux de plaie peropératoires élevés (36-37\%) (11,12). Dans la série de Peyronnet et al. (11) : 2 patientes sur 8 ont eu une plaie vaginale (25\%) et 1 une sur 8 , une plaie du col vésical $(12,5 \%)$. Cependant, dans la technique décrite dans cette série, la phase de dissection péricervicale, est réalisée en laparoscopie conventionnelle, et non en assistance robotique. Dans la série de Biardeau et al. $36 \%$ de complications per opératoires sont rapportées (2 plaies vaginales et 2 plaies vésicales) (12). Le siège de la dissection pourrait être différent, plus bas sur l'urètre, comme le suggère la taille des manchettes utilisées en comparaison à notre série $(6,5$ vs $7,7 \mathrm{~cm})$. On peut également avancer l'hypothèse que, l'ouverture du dôme vésical, très fréquente dans notre série $(11 / 14$, 78\%), ait réduit le risque de plaie rétrocervicale dans les cas difficiles multiopérés. En effet, dans notre groupe de primo-implantation, aucune plaie vésicale n'est à déplorer. En revanche, cela conduit à un sondage plus long, une durée d'hospitalisation plus longue et à un risque infectieux théorique. L'implantation de SUA par chirurgie robot assistée est en cours d'évaluation, et nous décrivons ici une technique de dissection. Pour ce qui concerne l'impact sur les complications peropératoires des différentes techniques de dissection décrites par voie robot assistée, il est trop tôt pour conclure et seule une étude comparative randomisée prospective serait valide.

Les résultats du SUA en matière de continence varient de 75 à $88 \%$ selon les séries et les voies d'abord $(4,6,8,11,12)$. Dans notre série les résultats sont bons et seules 2 patientes n'étaient pas continentes après l'implantation. La première a présenté un syndrome d'hyperactivité vésicale avec incontinence urinaire traité par stimulation du nerf tibial postérieur et porte une protection par jour au dernier suivi (patiente 3). 
Pour la seconde patiente, une révision de manchette a été réalisée 6 mois plus tard (taille 8 initialement, changée par une 6,5). Cette patiente a présenté une hyperactivité vésicale de novo actuellement contrôlée par anticholinergiques (patiente 12).

En dehors de l'installation du robot, la durée opératoire peut être prolongée en raison de temps d'adhésiolyse importants chez ces patientes multiopérées. Par ailleurs 4 patientes ont fait l'objet d'une promontofixation contemporaine du SUA, ce qui allongeait en moyenne l'intervention de 38 min (tableau 2), et explique les temps opératoires plus court rapportés par Biardeau et al., lesquels n'ont pas fait de geste associé. Nous rapportons ici le temps de dissection péricervical (46 min en moyenne ; Tableau 2) , pris entre le moment où débute l'incision du fascia endopelvien et celui ou on positionne le mesureur, qui nous semble être un moyen plus homogène d'évaluation de la durée du geste opératoire. Ce temps de dissection est variable et dépends des antécédents de dissection du col vésical.

En complément de notre précédente publication (6), nous présentons ici nos résultats sur 3 révisions. Sur ce nombre de cas limité, la procédure est donc réalisable par l'assistance robotique. Trolliet (10) a publié 8 cas de révision sur une série d'implantation par laparoscopie conventionnelle. Les durées, opératoire de sondage et d'hospitalisation étaient comparables aux nôtres, respectivement 120 VS 114 min ; 3 jours VS 2 jours et 5 jours VS 6 jours, et le taux de complication per et postopératoire nul. Lors des 3 révisions que nous avons pratiquées, les manchettes étaient placées au même site, avec une taille plus petite. Notons qu'il s'agit d'une procédure plus simple techniquement que les primo-implantations. En effet il n'y a pas de nouvelle dissection du col vésical en cas de remplacement pour matériel défectueux. Le bénéfice de 
l'assistance robotique dans ce cas nous est apparu moins grand que pour les révisions effectuées par cœlioscopie classique.

\section{Conclusion}

Nos résultats confirment la faisabilité de l'implantation, et de la révision d'un SUA par voie laparoscopique robot-assistée. Avec la réserve d'un nombre de cas limités et d'une étude monocentrique, le faible taux de complications et les résultats sur la continence sont encourageants. Néanmoins, l'expérience d'autres centres sera nécessaire pour confirmer la reproductibilité de la technique, et sa place par rapport aux approches en chirurgie ouverte et en cœlioscopie conventionnelle.

\section{Bibliographie}

1. Lucas MG, Bosch RJL, Burkhard FC, Cruz F, Madden TB, Nambiar AK, et al. EAU guidelines on surgical treatment of urinary incontinence. Eur Urol. 2012 Dec;62(6):1118-29. 2. Costa P, Mottet N, Rabut B, Thuret R, Ben Naoum K, Wagner L. The use of an artificial urinary sphincter in women with type III incontinence and a negative Marshall test. $\mathbf{J}$ Urol. 2001 Apr;165(4):1172-6.

3. Phé V, Benadiba S, Rouprêt M, Granger B, Richard F, Chartier-Kastler E. Long-term functional outcomes after artificial urinary sphincter implantation in women with stress urinary incontinence. BJU Int. 2014 Jun;113(6):961-7.

4. Costa P, Poinas G, Ben Naoum K, Bouzoubaa K, Wagner L, Soustelle L, et al. Longterm results of artificial urinary sphincter for women with type III stress urinary incontinence. Eur Urol. 2013 Apr;63(4):753-8.

5. Ngninkeu BN, van Heugen G, di Gregorio M, Debie B, Evans A. Laparoscopic Artificial Urinary Sphincter in Women for Type III Incontinence: Preliminary Results. Eur Urol. 2005 Jun;47(6):793-7.

6. Fournier G, Callerot P, Thoulouzan M, Valeri A, Perrouin-Verbe M-A. Roboticassisted Laparoscopic Implantation of Artificial Urinary Sphincter in Women With Intrinsic Sphincter Deficiency Incontinence: Initial Results. Urology. 2014 Nov;84(5):1094-8.

7. Abrams P, Andersson KE, Birder L, Brubaker L, Cardozo L, Chapple C, et al. Fourth International Consultation on Incontinence Recommendations of the International Scientific Committee: Evaluation and treatment of urinary incontinence, pelvic organ prolapse, and fecal incontinence. Neurourol Urodyn. 2010;29(1):213-40.

8. Ferreira C, Brychaert P-E, Menard J, Mandron E. Laparoscopic implantation of artificial urinary sphincter in women with intrinsic sphincter deficiency: Mid-term outcomes. Int J Urol. 2017 Apr 1;24(4):308-13.

9. Rouprêt M, Misraï V, Vaessen C, Cardot V, Cour F, Richard F, et al. Laparoscopic approach for artificial urinary sphincter implantation in women with intrinsic sphincter 
deficiency incontinence: a single-centre preliminary experience. Eur Urol. 2010 Mar;57(3):499-504.

10. Trolliet S, Mandron E, Lang H, Jacqmin D, Saussine C. Implantation de sphincter artificiel urinaire par voie laparoscopique chez des femmes avec incontinence urinaire d'effort sévère. Prog En Urol. 2013 Sep;23(10):877-83.

11. Peyronnet B, Vincendeau S, Tondut L, Bensalah K, Damphousse M, Manunta A. Artificial urinary sphincter implantation in women with stress urinary incontinence:

preliminary comparison of robot-assisted and open approaches. Int Urogynecology J. 2016 Mar;27(3):475-81.

12. Biardeau X, Rizk J, Marcelli F, Flamand V. Robot-assisted laparoscopic approach for artificial urinary sphincter implantation in 11 women with urinary stress incontinence: surgical technique and initial experience. Eur Urol. 2015 May;67(5):937-42. 


\section{Légendes}

Tableau 1 : Caractéristiques des patientes.

Table 1 : Patient characteristics.

Tableau 2 : Résultats du groupe Primo-Implantation.

Table 2 : Perioperative and functional results : Primo-implantation group.

Tableau 3 : Résultats du groupe révision.

Table 3 : Perioperative and functional results : revision group

Figure 1 : Disposition des bras du robot da Vinci SI ®.

Figure 1 : Positioning of the robot da Vinci SI ®.

Figure 2 : Dissection postérieure du col vésical par la pince Prograsp $®$ après ouverture vésicale.

Figure 2 : Posterior dissection of the bladder neck completed with the Prograsp forceps in place and opened bladder. 


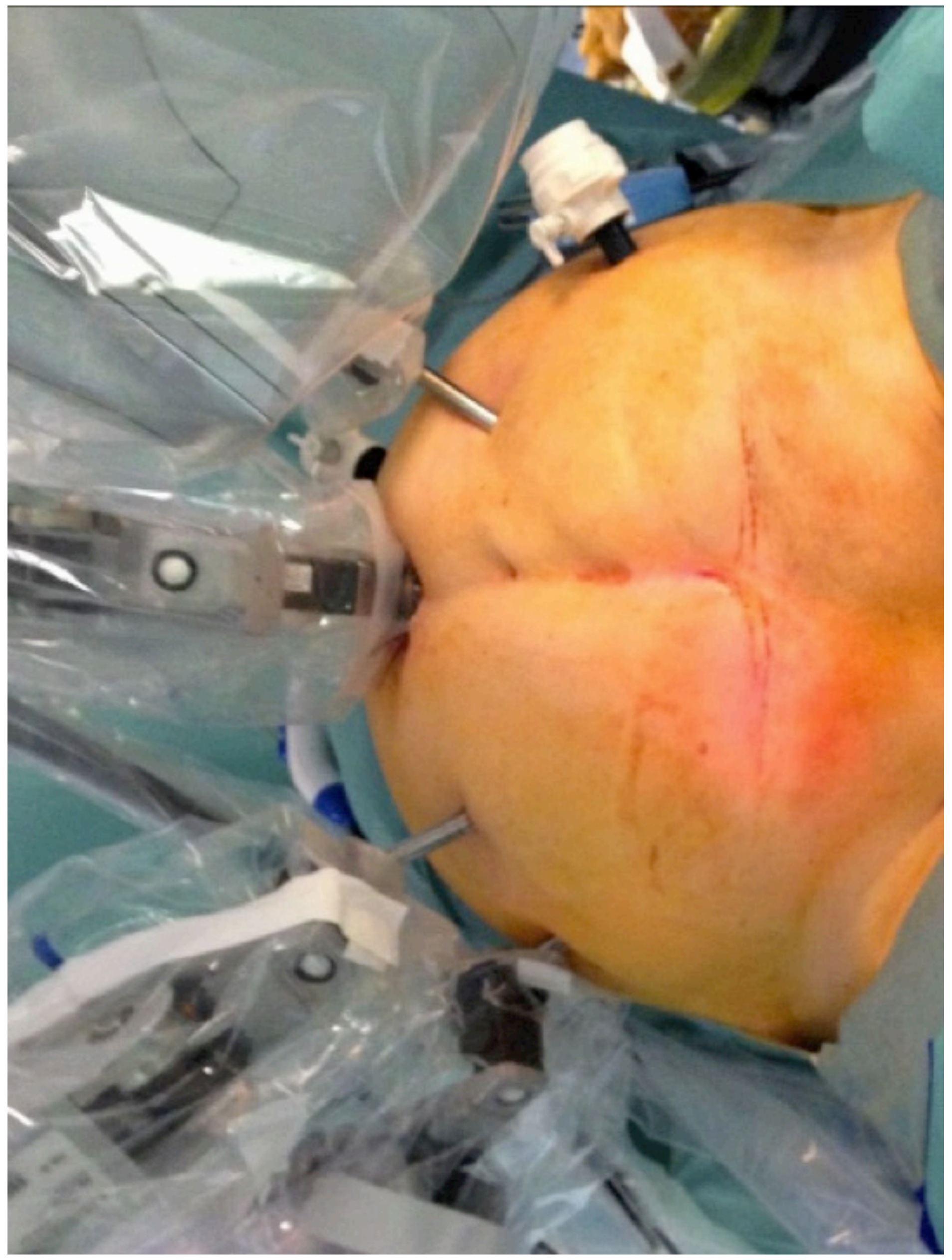




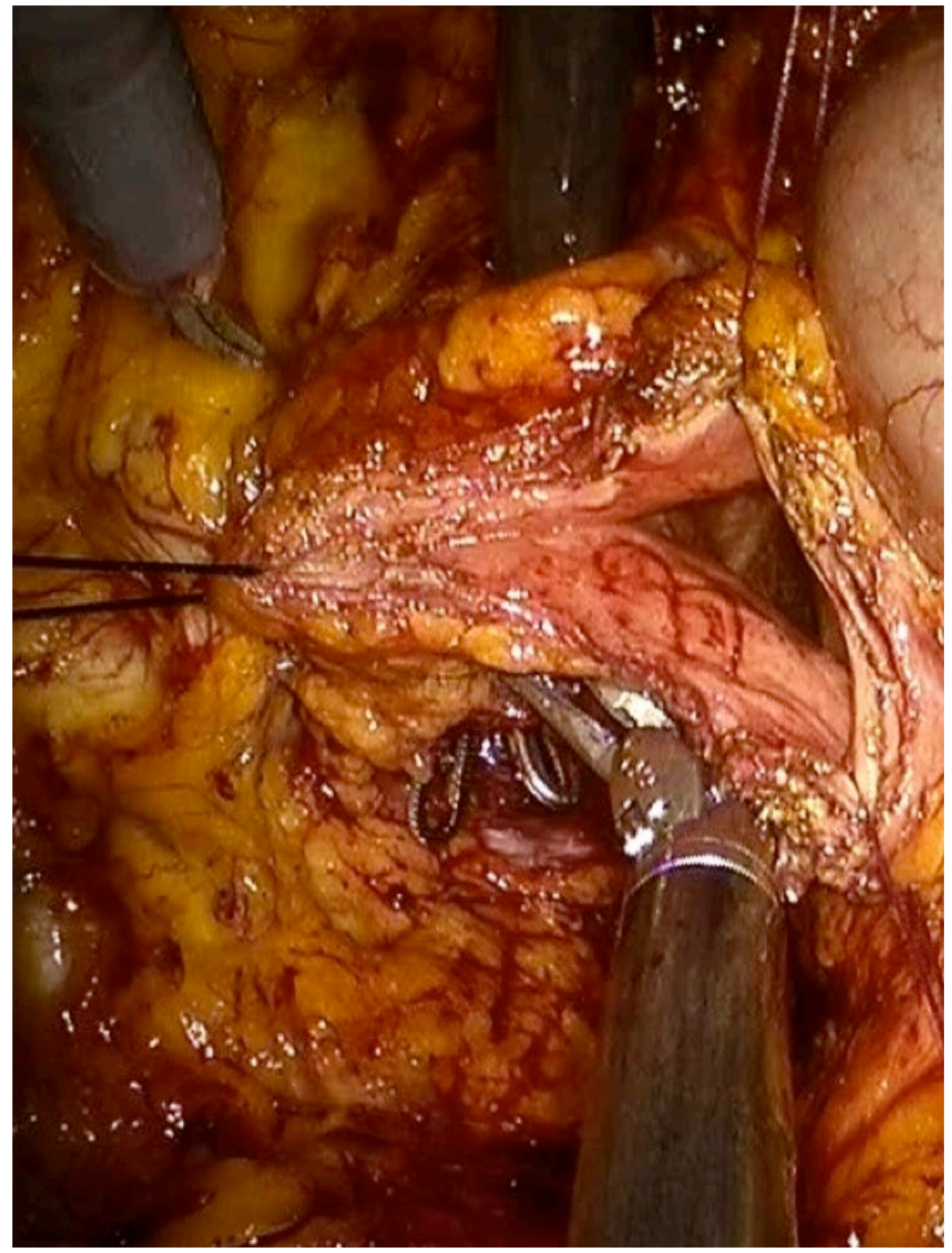




\begin{tabular}{|c|c|c|c|c|c|c|c|c|c|c|c|c|c|c|c|}
\hline \multirow[b]{3}{*}{ Caractéristique } & \multirow{3}{*}{ Population * } & \multicolumn{14}{|c|}{ Tableau 1a Caractéristiques des patientes primo-implantées. } \\
\hline & & \multicolumn{14}{|c|}{ Patientes } \\
\hline & & 1 & 2 & 3 & 4 & 5 & 6 & 7 & 8 & 9 & 10 & 11 & 12 & 13 & 14 \\
\hline Age (années) & $66,8 \pm 7$ & 75 & 66 & 74 & 58 & 50 & 67 & 74 & 68 & 63 & 72 & 65 & 62 & 69 & 72 \\
\hline IMC $(\mathrm{kg} / \mathrm{m} 2)$ & $26+/-5$ & 31 & 23 & 23 & 25 & 38 & 34 & 23 & 27 & 30 & 24 & 23 & 24 & 20 & 27 \\
\hline PCU $(\mathrm{cmH} 20)$ & $19,8 \pm 7,4$ & 17 & 30 & 30 & - & 15 & 11 & 13 & 20 & 15 & 28 & 23 & 20 & 33 & 12 \\
\hline Fuites (protections / j) & $4 \pm 2$ & 3 & 4 & 4 & Sonde & 7 & 3 & 10 & 4 & 5 & 6 & 3 & 3 & 2 & 3 \\
\hline $\begin{array}{l}\text { Antécédent de chirurgie de } \\
\text { l'incontinence }\end{array}$ & $1,8 \pm 1,9$ & 1 & 3 & 1 & 3 & 0 & 1 & 4 & 1 & 1 & 0 & 2 & 1 & 1 & 1 \\
\hline TVT & $8(57 \%)$ & - & + & + & + & - & - & + & + & + & - & + & + & - & - \\
\hline TVT-O & $5(36 \%)$ & - & + & - & - & - & + & + & - & - & - & + & - & + & - \\
\hline $\begin{array}{l}\text { Section BSU pour érosion } \\
\text { urétrale }\end{array}$ & $3(21 \%)$ & - & + & - & + & - & - & + & - & - & - & - & - & - & - \\
\hline Burch & $2(14 \%)$ & + & - & - & - & - & - & - & - & - & - & - & - & - & + \\
\hline Marshall Marchetti & $1(7 \%)$ & - & - & - & - & - & - & + & - & - & - & - & - & - & - \\
\hline Ablation de SUA & $1(7 \%)$ & - & - & - & + & - & - & - & - & - & - & - & - & - & - \\
\hline Autre chirurgie pelvienne & $1 \pm 0,7$ & 1 & 1 & 0 & 0 & 0 & 1 & 1 & 0 & 0 & 0 & 1 & 1 & 0 & 1 \\
\hline $\begin{array}{l}\text { Promontofixation } \\
\text { coelioscopique }\end{array}$ & $4(28 \%)$ & + & + & - & - & - & - & + & - & - & - & + & - & - & - \\
\hline Voie basse autologue & $3(21 \%)$ & - & - & - & - & - & + & - & - & - & - & - & + & - & + \\
\hline
\end{tabular}




\begin{tabular}{|c|c|c|c|c|}
\hline \multirow[b]{2}{*}{ Caractéristique } & \multirow[t]{2}{*}{ Population* } & \multicolumn{3}{|c|}{ Patientes } \\
\hline & & 1 & 2 & 3 \\
\hline Age (années) & $72,7 \pm 5$ & 68 & 78 & 72 \\
\hline IMC $(\mathrm{kg} / \mathrm{m} 2)$ & $25 \pm 8$ & 20 & 34 & 21 \\
\hline Age du SUA remplacé (années) & $15,3 \pm 1,5$ & 15 & 17 & 14 \\
\hline $\begin{array}{l}\text { Antécédent chirurgical (en dehors du } \\
\text { SUA) }\end{array}$ & $2,7 \pm 1,1$ & 2 & 2 & 4 \\
\hline TVT & 0 & - & - & - \\
\hline TVT-O & 0 & - & - & - \\
\hline Burch & $3(100 \%)$ & + & + & + \\
\hline Fronde sous cervicale & $1(33,3 \%)$ & - & - & + \\
\hline Ablation de SUA & $1(33,3 \%)$ & - & - & + \\
\hline Promontofixation coelioscopique & $3(100 \%)$ & + & + & + \\
\hline \multicolumn{5}{|c|}{$\begin{array}{l}\text { IMC : Indice de masse corporelle ; PCU : pression de clôture urétrale maximale } \\
\text { SUA : sphincter urinaire artificiel. } \\
\text { *Les données sont représentées par la moyenne } \pm \sigma \text { ou par le nombre } \mathrm{n}(\%) \text {. }\end{array}$} \\
\hline
\end{tabular}




\begin{tabular}{|c|c|c|c|c|c|c|c|c|c|c|c|c|c|c|c|}
\hline \multirow[t]{2}{*}{$\begin{array}{l}\text { Variable } \\
\text { nat }\end{array}$} & \multirow[t]{2}{*}{ Population } & \multicolumn{14}{|c|}{ Patientes } \\
\hline & & 1 & 2 & 3 & 4 & 5 & 6 & 7 & 8 & 9 & 10 & 11 & 12 & 13 & 14 \\
\hline Durée d'intervention* (min) & $205 \pm 34$ & 240 & 240 & 180 & 240 & 180 & 180 & 210 & 190 & 140 & 210 & 200 & 270 & 210 & 180 \\
\hline $\begin{array}{l}\text { Promontofixation antérieure } \\
\text { associée }\end{array}$ & $4(28,6 \%)$ & + & - & - & - & - & - & - & - & - & + & - & + & + & - \\
\hline Ouverture vésicale & $11(78,6 \%)$ & - & - & + & + & + & + & + & + & + & - & + & + & + & + \\
\hline Plaie vaginale & $1(7,1 \%)$ & - & - & - & - & - & - & - & - & - & - & - & - & - & + \\
\hline Plaie urétrale & 0 & - & - & - & - & - & - & - & - & - & - & - & - & - & - \\
\hline Plaie vésicale** & 0 & - & - & - & - & - & - & - & - & - & - & - & - & - & - \\
\hline Complication post opératoire & $3(21,4 \%)$ & - & - & - & - & - & - & - & - & - & - & - & - & - & - \\
\hline Hématome périnéal & 1 & - & - & - & + & - & - & - & - & - & - & - & - & - & - \\
\hline Rétention urinaire & 2 & - & - & - & - & - & - & - & + & - & - & - & - & + & - \\
\hline $\begin{array}{l}\text { Durée de dissection du col } \\
\text { (min) }\end{array}$ & $46 \pm 21$ & 60 & 60 & - & 90 & 70 & 50 & 40 & 25 & 22 & 30 & - & 25 & 40 & 42 \\
\hline Taille des manchettes $(\mathrm{cm})$ & $7,7 \pm 0,9$ & 7,5 & 7 & 7,5 & 9 & 8 & 9 & 9 & 7 & 8 & 7 & 8 & 8 & 6,5 & 6,5 \\
\hline Durée de sondage (jours) & $5,9 \pm 2,1$ & 2 & 2 & 7 & 7 & 7 & 7 & 7 & 7 & 7 & 2 & 7 & 7 & 7 & 7 \\
\hline Durée d'hospitalisation (jours) & $6,6 \pm 1,5$ & 4 & 5 & 8 & 6 & 6 & 9 & 9 & 7 & 5 & 6 & 5 & 7 & 8 & 7 \\
\hline Durée de suivi (mois) & $24,6 \pm 18,4$ & 39,9 & 46,2 & 60,1 & 47 & 36,8 & 9,5 & 3,8 & 16,2 & 4,9 & 16,9 & 22,9 & 19,3 & 4,5 & 14,3 \\
\hline $\begin{array}{l}\text { Continence totale } \\
\text { (nb protection/j) }\end{array}$ & $12(86 \%)$ & 0 & 0 & 1 & 0 & 0 & 0 & 0 & 0 & 0 & 0 & 0 & 3 & 0 & 0 \\
\hline Hyperactivité vésicale de novo & 4 & - & - & + & - & - & - & - & - & + & + & - & + & - & - \\
\hline
\end{tabular}


Tableau 3 Résultats du groupe révision (R).

\begin{tabular}{|c|c|c|c|c|}
\hline \multirow[t]{2}{*}{ Variable } & \multirow[t]{2}{*}{ Population } & \multicolumn{2}{|c|}{ Patientes } & \\
\hline & & 15 & 16 & 17 \\
\hline Durée d'intervention (min) & $112 \pm 8$ & 110 & 120 & 105 \\
\hline Complication post opératoire Clavien I & $1(33 \%)$ & - & - & + \\
\hline Taille des manchettes $(\mathrm{cm})$ & $5,2 \pm 0,8$ & 4,5 & 6 & 5 \\
\hline Durée de sondage (jours) & $4,3 \pm 4$ & 2 & 2 & 9 \\
\hline Durée d'hospitalisation (jours) & $7 \pm 3,6$ & 6 & 4 & 11 \\
\hline Durée de suivi (mois) & $58,2 \pm 5,2$ & 64 & 56 & 55 \\
\hline Continence (nb protections/j) & 0 & 0 & 0 & 0 \\
\hline
\end{tabular}

